\title{
Diferencias de metas deportivas entre estudiantes de educación física y deportistas: análisis de la invarianza del modelo entre ellos*
}

\section{Differences of Sport Goals between Students of Physical Education and Sportsmen: Analysis of the Invariance of Model among them}

\author{
Leandro Navas Martínez** \\ Universidad de Alicante, España \\ José Antonio Soriano Llorca*** \\ Colegio Nuestra Señora de Los Dolores, España \\ Francisco Pablo Holgado Tello**** \\ Universidad Nacional de Educación a Distancia, España \\ Manuela López Núñez***** \\ Colegio Público León Felipe, España
}

\footnotetext{
**Departamento de Psicología Evolutiva y Didáctica, Facultad de Educación. Profesor Titular de Psicología Evolutiva y de la Educación. Doctor. Correo electrónico: Leandro.Navas@ua.es

***Profesor de Enseñanza Secundaria. Doctor. Correo electrónico: jantonio_soriano@hotmail.com

*****Departamento de Metodología de las Ciencias del Comportamiento, Facultad de Psicología. Profesor Titular de Psicometría. Doctor. Correo electrónico: pfholgado@psi.uned.es

*****Doctora en Psicopedagogía. Directora de Colegio Infantil y de Primaria. Correo electrónico:mlnuyez@yahoo.es
}

\section{RESUMEN}

El objetivo de este estudio es averiguar si hay diferencias en las metas deportivas en función de que la actividad física se realice en contextos escolares o de competiciones deportivas. Participan 574 estudiantes de Educación Secundaria Obligatoria, entre los 11 y 17 años, y 511 deportistas, entre los 16 y 45 años, quienes responden al Cuestionario de Orientación a la Tarea y al Ego en el Deporte. Los resultados permiten confirmar el modelo teórico y muestran que, tanto en las orientaciones de meta a la tarea y al ego, las puntuaciones medias resultan más elevadas en el caso de los deportistas. Mediante análisis factorial confirmatorio multigrupo se encuentran algunas diferencias relevantes que son discutidas.

Palabras clave

educación física, deporte, orientación a la tarea, orientación al ego.

\begin{abstract}
The purpose of this study is to determine if there are differences in the function of sports goals whether the physical activity is done in school settings or competitions. Five hundred and seventy-four students of Obligatory Secondary Education took part on this study, aged between 11 and 17, and 511 athletes, aged between 16 and 45 years, who answer to the Task and Ego Orientation in Sport Questionnaire. The results confirm the theoretical model and show that the average scores are higher in the case of athletes, in both cases goal orientations to the task and ego. Through a confirmatory multi group factorial analysis some relevant differences are found that have been discussed.
\end{abstract}

Keywords

physical education, sport, task orientation, ego orientation. 
Para citar este artículo: Navas Martínez, L., Soriano Llorca, J.A., Holgado Tello, F.P., López Núñez, M. (2016). Diferencias de metas deportivas entre estudiantes de educación física y deportistas: análisis de la invarianza del modelo entre ellos. Universitas Psychologica, 15(4). http://dx.doi.org/10.11144/Javeriana.ups y15-4.mdee

Este trabajo se interesa por la motivación para la actividad física y parte de la teoría de las perspectivas o de las orientaciones de meta que es una de las más empleadas en la comprensión de las variables relacionadas con la motivación de logro (Duda, Fox, Biddle, \& Amstrong, 1992; Duda \& Nicholls, 1992; Duda, Olson, \& Templin, 1991; Roberts, 2001) y que muestra la existencia de dos orientaciones de meta en los deportistas: a la tarea y al ego.

La meta a la tarea consiste en demostrar aprendizaje y dirige la conducta cuando el aprendizaje o la maestría son muy importantes. El éxito depende de la valoración subjetiva de si se aprendió o se mejoró. Por contra, la meta al ego se relaciona más con la competitividad, consiste en maximizar la probabilidad de atribuirse alta habilidad a uno mismo y se hace patente cuando se da una intensa comparación social: el éxito depende de la valoración subjetiva que resulta de comparar la habilidad propia con la de otros (Castillo, Balaguer, \& Duda, 2002; García-Calvo, Leo, Martín, \& Sánchez, 2008; Holgado, Navas, López, \& García-Calvo, 2010; Núñez, Martín-Albo, Paredes, Rodríguez, \& Chipana, 2011; Valle et al., 2010).

La relevancia de estas orientaciones de meta en el deporte y que se asocian a cogniciones y a comportamientos diferentes ha quedado demostrada (Balaguer, Castillo, Duda, \& GarcíaMerita, 2011; Baser, Bayar, \& Ghorbanzadeh, 2013; Carlin, Salguero, Márquez, \& Garcés, 2009; Carr, 2012; Cecchini, González, \& Montero, 2008; Chin, Khoo \& Low, 2012; García-Calvo, Santos-Rosa, Jiménez \& Cervelló,
2006; García-Mas \& Gimeno, 2008; Holgado, Navas, \& López, 2010; Modroño \& Guillén, 2016; Roberts, 2001; Ruiz \& Piéron, 2013; Sage \& Kavussanu, 2007; Saotome, Harada, \& Nakamura, 2012; Zarauz \& Ruiz-Juan, 2014; Zubiaur \& Del Riego, 2015), de modo que los sujetos orientados a la tarea atribuyen sus éxitos internamente, perciben el deporte como una actividad que fortalece la capacidad de cooperación y el compromiso, que incrementa el interés por aprender y por mejorar, por cumplir las reglas, por esforzarse y por mejorar la salud y las habilidades deportivas, demostrando estar más satisfechos con su rendimiento y disfrutando más del deporte, con una conducta más adaptativa y que fomenta el juego limpio. Todo ello da lugar a una participación más entusiasta. Por otra parte, los sujetos orientados hacia el ego realizan atribuciones externas para los resultados, perciben la actividad como un sistema de mejorar la vida social, de adquirir un mayor reconocimiento social y popularidad y conseguir riqueza económica. Ello supone una participación menos entusiasta y mayor tendencia a desvincularse de la práctica deportiva.

Por otro lado, diversos estudios centrados en el contexto educativo, encontraron que la orientación de meta hacia la tarea es la que correlaciona poderosamente con mediadores positivos (Baena, Granero, Gómez, \& Abraldes, 2014; Baena-Extremera \& Ruiz-Juan, 2015; Bortoli, Bertollo, Comania, \& Robazza, 2011; Castillo-Andrés, Campos-Mesa, \& Ries, 2013; Jiménez, Cervelló, García, Santos-Rosa, \& Del Villar, 2006; Ruiz \& Piéron, 2013) y con mejores niveles de rendimiento escolar (Alemán, Trías, \& Curione, 2011; Barca, Peralbo, Porto, Marcos, \& Brenlla, 2011).

Así mismo, también hay que resaltar dos aspectos importantes de las orientaciones de meta, el primero de ellos es su carácter ortogonal, es decir, se pueden dar al mismo tiempo cierto grado de ambas orientaciones, conformando perfiles motivaciones (AlonsoTapia, Huertas, \& Ruiz, 2010; Manzano \& Valero, 2013; Navas, Soriano, Holgado, \& López, 2009; Saies, Arribas-Galarrag, Cecchini, 
Luis-De-Cos, \& Otaegui, 2014; Schwinger \& Stiensmeier-Pelster, 2011; Wosnitza \& Volet, 2012), y el segundo, que existen estudios en los que la orientación de meta al ego sí tiene una contribución positiva hacia la cantidad de práctica, el conceder importancia a la variedad en los ejercicios, el nivel deportivo y la disciplina (García-Mas \& Gimeno, 2008; Guzmán \& García-Ferriol, 2002), por ello, es importante promover la implicación hacia las orientaciones de meta pero sin especificar el carácter negativo de las orientaciones hacia el ego.

Todo lo expuesto permite plantear si el hecho de que la actividad física se realice en contextos escolares, dentro de la clase de Educación Física, o en ámbitos de competiciones deportivas introduce diferencias en las orientaciones de meta, ya que al variar el contexto y los fines de la actividad física, podrían variar igualmente las metas perseguidas. Téngase en cuenta que los objetivos de las clases de Educación Física distan mucho de los establecidos para el deporte de competición (Cervelló \& Santos-Rosa, 2000; Navas, Soriano, \& Holgado, 2006) o cuando este se realiza con otras finalidades (ocio o estar en forma). Por todo ello, el objetivo de este estudio es averiguar si se producen diferencias en las orientaciones de meta en función del contexto (el escolar o el de competición) en el que se realiza la actividad física o el deporte.

Como hipótesis puede aventurarse que las puntuaciones en la orientación de meta a la tarea serán más elevadas en el contexto escolar porque en él se enfatiza el aprendizaje y, al contrario, las puntuaciones en la orientación de meta al ego serán más elevadas en el contexto de competición deportiva que enfatiza más la competitividad.

\section{Método}

\section{Participantes}

Participan 574 estudiantes de los cuatro cursos de ESO, seleccionados por muestreo aleatorio simple, de ellos el $26.8 \%$ cursa $1^{\circ}$ de ESO, el $23.3 \%$ está en $2^{\circ}$, el $26.1 \%$ cursa $3^{\circ}$ y el $23.7 \%$ restante corresponde a $4^{\circ}$, todos ellos pertenecientes a la provincia de Alicante, y 511 deportistas federados que practicaban diversos deportes (baloncesto, balonmano, rugby, atletismo, tenis, kárate, natación, ciclismo, gimnasia rítmica, tiro con arco, etc.) un $49.7 \%$ realizaban deportes de equipo y un $50.3 \%$ deportes individuales, seleccionados por muestreo incidental, pertenecientes a diversas provincias (Alicante, Valencia, Bilbao, La Coruña, Granada, etc.). El número total de participantes es 1085. Las edades de los sujetos oscilan entre 11 y 45 años ( $M=17.99 ; D T=$ 5.91). El $61 \%$ son hombres y el $39 \%$ mujeres.

\section{Instrumentos}

Se emplea para evaluar las metas de los sujetos el TEOSQ (Cuestionario de Orientación a la Tarea y al Ego en el Deporte; Duda, 1989) en la versión de Balaguer, Castillo, y Tomás (1996). La consistencia interna (alfa de Cronbach) de las escalas ego y tarea es de 0.84 y de 0.87 respectivamente.

\section{Variables}

Se consideran dos variables. Por una parte, las orientaciones de meta en el deporte y, por otro lado, el contexto en el que se lleva a cabo la actividad física. Dentro de las orientaciones de meta para el deporte están la orientación al ego (cuya puntuación se obtiene sumando las puntuaciones de los ítems 1, 3, 4, 6, 9 y 11 del cuestionario) y la orientación a la tarea (cuya puntuación equivale a la suma de las puntuaciones de los elementos 2, 5, 7, 8, 10, 12 y 13 del cuestionario). En cuanto a la variable contexto en el que se realiza la actividad física, se consideran dos: el escolar (cuando la actividad se desarrolla durante la clase de Educación Física) y el deportivo (cuando la actividad se lleva a cabo en situaciones de competición deportiva no escolar). 


\section{Procedimiento}

Los sujetos respondieron al cuestionario en situaciones naturales (en el aula habitual los estudiantes $y$ al concluir el entrenamiento los deportistas), fueron instruidos sobre la importancia de su sinceridad al responder y se les garantizó el anonimato. Así mismo, se solicitó permiso a los progenitores de los participantes menores de edad implicados, y se les informó sobre cuál iba a ser la posterior utilización de los datos.

\section{Diseño y análisis de datos}

Al no haber manipulación intencional de las variables, el diseño es del tipo correlacional básico. Se realizaron análisis factoriales exploratorio (AFE) y confirmatorio (AFC) y el contraste de diferencias de medias (prueba $t$ de Student). Para realizar el AFC se utiliza la matriz de correlaciones policóricas, pues los ítems se consideran variables continuas latentes que han sido categorizadas. Bajo estas condiciones, el método de estimación recomendado es mínimos cuadrados ponderados (WLS) ya que para muestras grandes y cuando no hay muchas variables proporciona estimadores consistentes, mejores índices de ajuste y pruebas chi-cuadrado (Flora \& Curran, 2004).

\section{Resultados}

Como el TEOSQ fue especialmente diseñado para evaluar las orientaciones de meta de las personas en el contexto deportivo, las respuestas de los participantes se sometieron a análisis factoriales (AFE y AFC) cuyos resultados vinieron a confirmar el modelo subyacente al cuestionario y que ha sido establecido consistentemente en la literatura (Castillo et al., 2002). Los valores obtenidos para los distintos índices de ajuste $\left(\lambda \mathrm{x}^{2}=295.50 ; \mathrm{gl}=64 ; \mathrm{p}\right.$ $<$

0.001; RMSEA $=0.06 ; \mathrm{GFI}=0.98 ; \mathrm{AGFI}=0.97$ ) permiten concluir que el modelo tiene un buen ajuste. Ningún índice de modificación mejora notablemente el modelo a nivel estadístico y dado el buen ajuste, tanto teórico como empírico, que presenta, tampoco se ha visto necesario añadir o modificar parámetros. En la Tabla 1 se ofrecen los coeficientes $\lambda \mathrm{x}_{\mathrm{x}}$ correspondientes a la solución completamente estandarizada.

\section{Tabla 1}

Solución completamente estandarizada del AFC para el TEOSQ

\begin{tabular}{lll}
\hline Items & Ego & Tarea \\
\hline 1. Soy el único que puede hacer la jug ada en cuestión & 0.69 & \\
3. Yo puedo hacerlo mejor que mis compañeros & 0.77 & \\
4. Mis compañeros no pueden hacerlo tan bien como yo & 0.84 & \\
6. Otros fallan y yo no & 0.79 & \\
9. Consigo más puntos, goles, etc. que todos & 0.84 & \\
11. Soy el mejor & 0.81 & \\
2. Aprendo una nueva habilidad y me impulsa a practicar más & & 0.70 \\
5. Aprendo algo que es divertido & 0.49 \\
7. Aprendo una nueva habilidad esforzándome mucho & 0.71 \\
8. Trabajo realmente duro durante la clase & 0.75 \\
10. Algo que he aprendido me impulsa a practicar más & 0.80 \\
12. Noto que una habilidad que he aprendido funciona & 0.69 \\
13. Pongo todo lo que está de mi parte (todo lo que puedo) & 0.60 \\
\hline Ego & 0.08 \\
\hline
\end{tabular}

Fuente: elaboración propia

Se realizó un contraste de medias (prueba $\mathrm{t}$ de Student) para muestras independientes, considerando como variable dicotómica de agrupamiento el contexto en el que se realiza la actividad física (escolar y de competición) y como variable agrupada las puntuaciones en las orientaciones de meta. La prueba de Levene para la igualdad de las varianzas poblacionales resulta estadísticamente significativa $(\mathrm{F}=30.56$; $\mathrm{p}<$ 0.001) en el caso de la orientación al ego y, en consecuencia, se asume que dichas varianzas son diferentes. 


\section{Tabla 2}

Prueba t de Student para las diferencias de medias considerando como variables dependientes las orientaciones de meta (al ego y a la tarea) y como variable independiente el contexto en el que se realiza la actividad física (escolar o deporte de competición)

\begin{tabular}{llccccc}
\hline & Variables & $N$ & $M$ & $t$ & $g l$ & $p$ \\
\hline Ego & Escolar & 574 & 15.636 & -5.01 & 977.2 & 0 \\
& $\begin{array}{l}\text { Deporte de } \\
\text { competición }\end{array}$ & 511 & 17.325 & & & \\
Tarea & Escolar & 574 & 28.223 & -4.462 & 1083 & 0 \\
& $\begin{array}{l}\text { Deporte de } \\
\text { competición }\end{array}$ & 511 & 29.292 & & & \\
\hline
\end{tabular}

Fuente: elaboración propia

Como se puede observar en la Tabla 2, las diferencias de medias son estadísticamente significativas. Muestran puntuaciones más elevadas en ambas orientaciones de meta quienes realizan la actividad física en contextos de competición.

Una vez analizada la bondad de ajuste del modelo propuesto con los datos obtenidos en la muestra total (véase la Tabla 1), se evaluó dicho ajuste del modelo en los dos grupos y se detectó qué ítems y factores fueron entendidos diferencialmente.

\section{Ajuste del modelo base en los dos grupos (estudiantes-deportistas)}

Para el grupo de estudiantes se obtiene un $\chi^{2}=$ $152.53(\mathrm{gl}=64 ; \mathrm{p}<0.001)$; RMSEA = 0.059; $\mathrm{GFI}=0.98 ; \mathrm{AGFI}=0.97 ; \mathrm{CFI}=0.94$. Para los deportistas se encuentra un $\chi^{2}=132.24(\mathrm{gl}=64 ; \mathrm{p}$ $=0) ;$ RMSEA $=0.052 ;$ GFI $=0.98 ;$ AGFI $=0.97$ y CFI $=0.94$. Ahora bien, observando los índices de modificación en el grupo de estudiantes se aprecia que al hacerse la inclusión del ítem 13 "pongo todo lo que está de mi parte" en el factor de orientación de meta al ego, el modelo mejora significativamente $\left(\mathrm{D} \chi^{2}=9.13 ; \mathrm{D} g l=1\right)$. La inclusión de dicho ítem se considera relevante porque se entiende que, al saturar negativamente, el no esforzarse al máximo podría caracterizar a la orientación al ego.
Las soluciones completamente estandarizadas para ambos grupos se muestran en la Tabla 3 . A simple vista se observa que los coeficientes lambda son diferentes entre ambos grupos al igual que la asociación entre los factores. A pesar de estas diferencias, con los resultados obtenidos se puede considerar que el modelo base representa los datos de manera adecuada en ambos grupos.

\section{Tabla 3}

Solución completamente estandarizada del AFC para los estudiantes y deportistas

\begin{tabular}{|c|c|c|c|c|}
\hline İtems & $\begin{array}{l}\text { Ego } \\
\text { Est. Dep. }\end{array}$ & $\begin{array}{c}\text { Tarea } \\
\text { Est. Dep. }\end{array}$ & & \\
\hline $\begin{array}{l}\text { 1. Soy el único que puede } \\
\text { hacer la jugada en cuestión }\end{array}$ & 0.66 & & & \\
\hline $\begin{array}{l}\text { 3. Yo puedo hacerlo mejor } \\
\text { que mis compañeros }\end{array}$ & 0.76 & & 0.78 & \\
\hline $\begin{array}{l}\text { 4. Mis compañeros no pueden } \\
\text { hacerlo tan bien como yo }\end{array}$ & 0.82 & & 0.84 & \\
\hline 6. Otros fallan y yo no & 0.75 & & 0.86 & \\
\hline $\begin{array}{l}\text { 9. Consigo más puntos, goles, } \\
\text { etc., que otros }\end{array}$ & 0.82 & & 0.87 & \\
\hline 11. Soy el mejor & 0.84 & 0.80 & & \\
\hline $\begin{array}{l}\text { 2. Aprendo una nueva } \\
\text { habilidad y me impulsa a } \\
\text { practicar más }\end{array}$ & & & 0.72 & 0.69 \\
\hline $\begin{array}{l}\text { 5. Aprendo algo que es } \\
\text { divertido }\end{array}$ & & & 0.58 & 0.5 \\
\hline $\begin{array}{l}\text { 7. Aprendo una nueva } \\
\text { habilidad esforzándome } \\
\text { mucho }\end{array}$ & & & 0.69 & 0.80 \\
\hline $\begin{array}{l}\text { 8. Trabajo realmente duro } \\
\text { durante la clase }\end{array}$ & & & 0.73 & 0.77 \\
\hline $\begin{array}{l}\text { 10. Algo que he aprendido me } \\
\text { impulsa a practicar más }\end{array}$ & & & 0.78 & 0.83 \\
\hline $\begin{array}{l}\text { 12. Noto que una habilidad } \\
\text { que he aprendido funciona }\end{array}$ & & & 0.66 & 0.72 \\
\hline $\begin{array}{l}\text { 13. Pongo todo lo que está de } \\
\text { mi parte (todo lo que puedo) }\end{array}$ & -0.14 & & 0.69 & 0.56 \\
\hline Ego & & & 0.17 & -0.5 \\
\hline
\end{tabular}

Fuente: elaboración propia

\section{Evaluación de la invarianza a través de la modalidad de práctica deportiva}

\section{Equivalencia de la estructura del modelo analizado}

En primer lugar, se realizó un análisis multigrupo, es decir, considerando ambos grupos conjuntamente $\mathrm{y}$, por lo tanto, estimando los parámetros simultáneamente sin imponer 
ninguna restricción sobre la equivalencia de la matriz de pesos factoriales, ni sobre la varianza/ covarianza de los factores, y se fijaron a uno los parámetros de los ítems 11 y 13. Los resultados obtenidos se muestran en la primera fila de la Tabla 4 (modelo 1 o de comparación).

Los índices de bondad de ajuste considerando simultáneamente ambos grupos (modelo 1) fueron $\chi^{2}=275.64(\mathrm{gl}=127 ; p<0.001)$; ECVI $=0.48 ;$ RMSEA $=0.054 ;$ GFI $=0.98$ y CFI $=$ 0.95 . Con estos resultados se puede aceptar la hipótesis de que el modelo compuesto por una variable latente de orientación de meta al ego y otra a la tarea existe tanto en deportistas como en estudiantes. A pesar de ello, no tiene por qué haber necesariamente un patrón común de las saturaciones factoriales entre ambos grupos. Por ello, y siguiendo a Byrne (1998) a continuación, se evalúa si las saturaciones de cada ítem son invariantes entre estudiantes y deportistas.

\section{Invarianza del modelo de las saturaciones factoriales}

Se parte de la hipótesis nula de que todos los coeficientes lambda son equivalentes en ambos grupos (Tabla 4, modelo 2). Si hubiera un incremento significativo en Chi-cuadrado en relación con el modelo 1, habría que ir probando la equivalencia ítem a ítem. Los resultados de la Tabla 4 muestran que la hipótesis de igualdad para los pesos factoriales no puede ser aceptada, dado que hay un incremento significativo en $\chi^{2}$ en relación con el modelo $1\left(\Delta \chi^{2}=42.58 ; \Delta g l=11\right)$. Por ello, se debe ir comprobando qué ítems están provocando dicho incremento significativo, lo que indicaría que estarían siendo entendidos distintamente por estudiantes y deportistas.

\section{Tabla 4}

Invarianza de las saturaciones factoriales del modelo de medida (modelo 2) entre estudiantes y deportistas

\begin{tabular}{|c|c|c|c|c|c|c|}
\hline Modelo & $\chi^{2}\left(\Delta \chi^{2}\right)$ & $g l(\Delta g l)$ & ECVI & RMSEA & GFI & CFI \\
\hline modelo 1 & 275.64 & 127 & 0.48 & 0.054 & 0.98 & 0.95 \\
\hline \multirow{2}{*}{$\begin{array}{l}\text { modelo2 } \\
\text { Items } \\
\text { Ego }\end{array}$} & $(42.58)^{*}$ & -11 & 0.48 & 0.055 & 0.98 & 0.95 \\
\hline & $1(19.4)^{*}$ & -1 & 0.48 & 0.055 & 0.98 & 0.95 \\
\hline & $3(17.48)^{*}$ & -1 & 0.47 & 0.055 & 0.98 & 0.95 \\
\hline & $4 \quad(17.99)^{*}$ & -1 & 0.47 & 0.055 & 0.98 & 0.95 \\
\hline & $6 \quad(26.72)^{*}$ & -1 & 0.48 & 0.057 & 0.98 & 0.95 \\
\hline & $9 \quad(20.54)^{*}$ & -1 & 0.48 & 0.056 & 0.98 & 0.95 \\
\hline \multirow{2}{*}{\multicolumn{7}{|c|}{$\begin{array}{l}\text { Ítems } \\
\text { Tarea }\end{array}$}} \\
\hline & & & & & & \\
\hline & $2(18.81)^{*}$ & -1 & 0.47 & 0.055 & 0.98 & 0.95 \\
\hline & $5 \quad(17.58)^{*}$ & -1 & 0.47 & 0.055 & 0.98 & 0.95 \\
\hline & $7 \quad(26.68)^{*}$ & -1 & 0.48 & 0.057 & 0.98 & 0.95 \\
\hline & $8(23.23)^{*}$ & -1 & 0.48 & 0.056 & 0.98 & 0.95 \\
\hline 10 & $0 \quad(23.18)^{*}$ & -1 & 0.48 & 0.056 & 0.98 & 0.95 \\
\hline 12 & $2 \quad(24.49)^{*}$ & -1 & 0.48 & 0.056 & 0.98 & 0.95 \\
\hline
\end{tabular}

Nota: $\Delta \chi^{2}=$ incremento en $\chi^{2}$ con respecto al modelo 1 , o modelo de comparación y $\Delta g l=$ diferencia en grados de libertad. *p $<0.05$ Fuente: elaboración propia

Al observar la Tabla 4, encontramos que todos los ítems presentan un incremento significativo, es decir, su comportamiento no puede considerarse equivalente entre ambos grupos.

\section{Invarianza de la matriz de varianzal covarianza de los factores}

El objetivo es analizar la equivalencia de las relaciones entre los constructos teóricos (Vanderberg \& Lance, 2000). Observando la Tabla 5 encontramos un modelo estructural variante entre ambos grupos. El incremento en Chi-cuadrado para cada una de las varianzas (ego y tarea) y covarianzas (ego-tarea) es significativo. Es decir, la relación que establecen entre sí ego y tarea es distinta entre deportistas y estudiantes.

\section{Discusión}

Los resultados permiten concluir que el modelo teórico del Cuestionario de Orientación al Ego y la Tarea en el Deporte tiene un buen ajuste 
Diferencias de metas deportivas entre estudiantes de educación física y deportistas: análisis ...

y obtiene confirmación empírica en los datos. Aunque este instrumento fue desarrollado para evaluar las orientaciones de meta en el deporte (Duda, 1989) los resultados indican que es igualmente válido para contextos escolares.

La meta a la tarea consiste en demostrar que se domina el aprendizaje de una tarea y en dirigir la conducta en situaciones en las que aprendizaje es lo importante. Para un sujeto con meta a la tarea, el éxito depende de la percepción subjetiva de que ha mejorado (Castillo et al., 2002; García-Calvo et al., 2008; Holgado, Navas \& López, 2010; Kavassanu \& Roberts, 2001; Nicholls, 1984a, 1984b; Núñez et al., 2011; Roberts, 2001) y en ese sentido se aventuraba que, en los contextos escolares, las puntuaciones en la orientación de meta a la tarea serían más elevadas. Del mismo modo, dado que la meta al ego está relacionada con la competitividad y se pone de manifiesto cuando hay una intensa comparación social y el éxito depende de la valoración de superar a los demás (Duda, 1989; Kavassanu \& Roberts, 2001; Nicholls, 1984a, 1984b; Núñez et al., 2011), se planteaba como hipótesis que las puntuaciones en la meta al ego serían más altas en el contexto de competición. Por una parte, los resultados obtenidos nos conducen a rechazar la primera hipótesis, ya que la puntuación media de la meta a la tarea en contextos escolares es inferior a la puntuación media en contextos de competición, en contra de lo que se suponía y, por ello, los sujetos en contextos deportivos parecen estar más orientados a la tarea que en contextos escolares, tal y como ocurre cuando se comparan las orientaciones de meta entre deportistas novatos y expertos (Saies et al., 2014). Por otra parte, los resultados permiten aceptar la segunda hipótesis ya que la puntuación media de la meta al ego en contextos deportivos es superior a la puntuación media de la meta al ego en contextos escolares, tal y como se pensaba.

Dadas las diferencias encontradas, se estudió si el modelo se mantenía invariante entre deportistas y estudiantes. Para ello, se utilizó el análisis factorial confirmatorio multigrupo que representa distintos grados de invarianza de los modelos estructurales y de medida. Los resultados indican que tanto los pesos factoriales como la relación entre los constructos planteados son variantes entre ambos grupos. De entre ellos, destaca que la relación entre las orientaciones al ego y a la tarea en los estudiantes es positiva, mientras que en los deportistas es nula. Estas diferencias podrían estar marcadas porque la competición a la que posiblemente se ven sometidos los deportistas provoca que su forma de entender las orientaciones de meta a la tarea y al ego sea distinta.

Estos resultados pueden interpretarse al considerar la autonomía percibida y la autodeterminación (Balaguer, Castillo \& Duda, 2008; Biddle, 2001; Cuevas, García-López, \& Contreras, 2015; Dias, Corte-Real, Barreiros, Brustad, \& Fonseca, 2015; Rivas et al., 2012; Sampeiro, Jiménez-Castuera, Lobato, Leyton \& Claver, 2016; Zarauz \& Ruiz-Juan, 2014). En clase, la actividad física se puede percibir como impuesta y no escogida voluntariamente, en tanto que en el contexto deportivo, el sujeto puede percibir autonomía, practica el deporte elegido y la actividad no se ve tan impuesta. Esta podría ser una explicación de que, en ambas orientaciones de meta, los deportistas alcancen puntuaciones más elevadas que los escolares. Otras explicaciones provienen del clima motivacional, de los hábitos de entrenamiento o de los climas sociales (Gutiérrez, 2014; Gutiérrez, Ruiz, \& López, 2011; Kavussanu \& Roberts, 1996; SánchezOliva, Leo, Sánchez-Miguel, Amado, \& GarcíaCalvo, 2010; Marques, Nonohay, Koller, Gauer, \& Cruz, 2015; Moreno-Murcia, Huéscar, \& Parra, 2013; Sampeiro et al., 2016; Troncoso, Burgos, \& López-Walle, 2015, Zarauz, RuizJuan, \& Flores-Allende, 2016) diferentes que generen los entrenadores y los profesores de Educación Física.

Como posibles investigaciones futuras, sería interesante estudiar las orientaciones de meta, en relación con otras variables como la práctica deportiva, el tiempo de práctica, el tipo de práctica, la imagen corporal, las metas sociales, el autoconcepto físico, etc., lo que permitirá conocer más y mejor las características de los diferentes perfiles motivacionales. 


\section{Referencias}

Alemán, M. J., Trías, D., \& Curione, K. (2011). Orientaciones motivacionales, rendimiento académico y género en estudiantes de bachillerato. Ciencias Psicológicas, 5 (2), 159-166.

Alonso-Tapia, J., Huertas, J. A., \& Ruiz, M. A. (2010). On the nature of motivational orientations: Implications of Assessed goals and gender differences for motivational goal theory. The Spanish Journal of Psychology, 13 (1), 232-243. http://dx.doi.org/10.1017/S11387 41600003814

Baena, A., Granero, A., Gómez, M., \& Abraldes, J. A. (2014). Orientaciones de Meta y Clima Motivacional según sexo y edad en Educación Física. Cultura, Ciencia y Deporte, 9 (26), 119-128.

Baena-Extremera, A., \& Ruiz-Juan, F. (2015). Predicción de las metas de logro en educación física a partir de la satisfacción, la motivación y las creencias de éxito en el deporte. Revista Iberoamericana de Psicología del Ejercicio y el Deporte, 10 (2), 193-203.

Balaguer, I., Castillo, I., Duda, J. L., \& García-Merita, M. (2011). Asociaciones entre la percepción del clima motivacional creado por el entrenador, orientaciones disposicionales de meta, regulaciones motivacionales y vitalidad subjetiva en jóvenes jugadoras de tenis. Revista de Psicología del Deporte, 20 (1), 133-148.

Balaguer, I., Castillo, I., \& Duda, J. L. (2008). Apoyo a la autonomía, satisfacción de las necesidades, motivación y bienestar en deportistas de competición: Un análisis de la teoría de la autodeterminación. Revista de Psicología del Deporte, 17 (1), 123-139.

Balaguer, I., Castillo, I., \& Tomás, I. (1996). Análisis de las propiedades psicométricas del cuestionario de Orientación al Ego y a la Tarea en el Deporte (TEOSQ) en su traducción al castellano. Psicológica, 17 , 77-81.
Barca, A., Peralbo, M., Porto, A. Marcos J.L., \& Brenlla J.C. (2011). Metas académicas del alumnado de Educación Secundaria Obligatoria (ESO) y Bachillerato con alto y bajo rendimiento escolar. Revista de Educación, 354, 341-368.

Baser, B., Bayar, P., \& Ghorbanzadeh, B. (2013). A determination of goal orientation in respect to the age categories and gender volleyball players in relation to their succcess. European Journal of Experimental Biology, 3 (1), 81-84.

Biddle, S. J. H. (2001). Enhacing motivation in physical education. En G. C. Roberts (Ed.), Advances in motivation in sport and exercise (pp. 101-127). Champaing, IL: Human Kinetics.

Bortoli, L., Bertollo, M., Comania, S., \& Robazza, C. (2011). Competence, achievement goals, motivational climate, and pleasant psychobiosocial states in youth sport. Journal of Sports Sciences, 29 (2), 171-180. http://dx.doi.org/10.1080/02 640414.2010.530675

Byrne, B. (1998). Structural Equation Modelling with LISREL, PRELIS and SIMPLIS: Basics concepts, applications, and programming . Londres, Inglaterra: Lawrence Erlbaum Associates.

Carlin, M., Salguero, A., Márquez, S., \& Garcés, E. J. (2009). Estudio de las orientaciones de meta en deportistas universitarios: Análisis del género, tipo de deporte y nivel competitivo. Revista Iberoamericana de Psicología del Ejercicio y el Deporte, 4 (2), 201-216.

Carr, S. (2012). High task/high ego oriented sudents` reasons for endorsing task and ego goals in the context of physical education. Applied psychology: An International Review, 61 , 540-563. http://dx.doi.org/10. 1111/j.1464-0597.2012.00505.x

Castillo-Andrés, Ó., Campos-Mesa, M. C., $\&$ Ries, F. (2013). Gender equality in physical education from the perspective of achievement goal theory. Journal of Sport and Health Research, 5 (1), 57-70. 
Castillo, I., Balaguer, I., \& Duda, J. L. (2002). Las perspectivas de meta de los adolescentes en el contexto deportivo. Psicothema, 14 (2), 280-287.

Cecchini, J. A., González, C., \& Montero, J. (2008). Participación en el deporte, orientación de meta y funcionamiento moral. Revista Latinoamericana de Psicología, 40 (3), 497-509.

Cervelló, E. M., \& Santos-Rosa, F. J. (2000). Motivación en las clases de educación física: un estudio de la perspectiva de las metas de logro en el contexto educativo. Revista de Psicología del Deporte, 9 , 51-70.

Chin, N. S., Khoo, S., \& Low, W. Y. (2012). Selfdetermination and goal orientation in track and field. Journal of Human Kinetics, 33 , 151-161. http://dx.doi.org/10.2478/v10078 $-012-0054-0$

Cuevas, R., García-López, L. M., \& Contreras, O. (2015). Influencia del modelo de Educación Deportiva en las necesidades psicológicas básicas. Cuadernos de Psicología del Deporte, 15 (2), 155-162.

Dias, C., Corte-Real, N., Barreiros, A., Brustad, R., \& Fonseca, A. M. (2015). ¿Cómo distinguir entre jóvenes atletas que tienen la intención de continuar practicando deporte de los que no piensan continuar haciéndolo? Cuadernos de Psicología del Deporte, 15 (3), 27-40.

Duda, J. L. (1989). Relationship between task and ego orientation and the perceived purpose of sport among high school athletes. Journal of Sport and Exercise Psychology, 11 , 318-335.

Duda, J. L., Fox, K. R., Biddle, S. J. H., \& Armstrong, N. (1992). Children's achievement goals and beliefs about success in Sport. British Journal of Educational Psychology, 62, 309-323. http://dx.doi.org/10.1111/j.20448279.1992.tb01025.x

Duda, J. L., \& Nicholls, J. G. (1992). Dimensions of achievement motivation in schoolwork and sport. Journal of Educational
Psychology, 84 (3), 290-299. http://dx.doi. org/10.1037/0022-0663.84.3.290

Duda, J. L., Olson, L., \& Templin, T. (1991). The relationship of task and ego orientation to sportsmanship attitudes and the perceived legitimacy of injurious acts. Research Quarterly for Exercise and Sport, 62 , 79-87. http://dx.doi.org/10.1080/02701367 .1991 .10607522

Flora, D. B., \& Curran, P. J. (2004). An empirical evaluation of alternative methods of estimation for confirmatory factor analysis with ordinal data. Psychological Methods, 9 , 466-491. http://dx.doi.org/10. 1037/1082-989X.9.4.466

García-Calvo, T., Leo, F. M., Martín, E., \& Sánchez, P. A. (2008). El compromiso deportivo y su relación con factores disposicionales y situacionales contextuales de la motivación. Revista Internacional de Ciencias del Deporte, 12 (4), 45-58.

García-Calvo, T., Santos-Rosa, F. J., Jiménez, R. C., \& Cervelló, E. (2006). El clima motivacional en las clases de Educación Física: una aproximación práctica desde la Teoría de Metas de Logro. Apuntes: Educación Física y Deportes, 81 , 21-28.

García-Mas, A., \& Gimeno, F. (2008). La teoría de orientación de metas y la enseñanza de la Educación Física: consideraciones prácticas. Revista Latinoamericana de Psicología, 40 (3), 511-522.

Gutiérrez, M. (2014). Relaciones entre el clima motivacional, las experiencias en educación física y la motivación extrínseca de los alumnos. Retos. Nuevas tendencias en Educación Física, Deporte y Recreación, 26, 9-14.

Gutiérrez, M., Ruiz, M. L., \& López, E. (2011). Clima motivacional en Educación Física: concordancia entre las percepciones de los alumnos y las de sus profesores. Revista de Psicología del Deporte, 20 (2), 321-335.

Guzmán, J. F., \& García-Ferriol, A. (2002). Orientación de meta de los entrenadores y metodología de entrenamiento: 
Leandro Navas Martínez, José Antonio Soriano Llorca, Francisco Pablo Holgado Tello, Manuela López Núñez.

implicaciones motivacionales. Revista Motricidad, 9 , 65-82.

Holgado, F. P., Navas, L., \& López, M. (2010). Goal orientations in sport: a causal model. European Journal of Education and Psychology, 3 (1), 19-32.

Holgado, F. P., Navas, L., López, M., \& GarcíaCalvo, T. (2010). A structural model of goal orientation in Sports: Personal and contextual variables. The Spanish Journal of Psyhcology, 13 (1), 256-265.

Jiménez, R., Cervelló, E. M., García, T., SantosRosa, F. J., \& Del Villar, F. (2006). Relaciones entre las metas de logro, la percepción del clima motivacional, la valoración de la educación física, la práctica deportiva extraescolar y el consumo de drogas en estudiantes de educación física. Revista Mexicana de Psicología, 23 (2), 253-265.

Kavussanu, M., \& Roberts, G. C. (2001). Moral functioning in sport. An achievement goal perspective. Journal of Sport and Exercise Psichology, 23 , 37-54.

Manzano, D., \& Valero, A. (2013). Análisis del perfil motivacional de diversos grupos de atletas y su repercusión en el nivel de satisfacción deportiva. Revista Euroamericana de Ciencias del Deporte, 2 (2), 9-19.

Marques, M., Nonohay, R., Koller, S., Gauer, G., \& Cruz, J. (2015). El estilo de comunicación del entrenador y la percepción del clima motivacional generado por los entrenadores y compañeros. Cuadernos de Psicología del Deporte, 15 (2), 47-53.

Modroño, C., \& Guillén, F. (2016). Motivation and self-concept in windsurfers: A study of professional and recreacional participants. Revista de Psicología del Deporte, 25 (1), 105-112.

Moreno-Murcia, J. A., Huéscar, E., \& Parra, N. (2013). Manipulación del clima motivacional en educación física para evitar el aburrimiento. Revista Mexicana de Psicología, 30 (2), 108-114.
Navas, L., Soriano, J. A., \& Holgado, F. P. (2006). Orientaciones de meta en las clases de Educación física: Un análisis centrado en la Educación Secundaria. Revista de Psicología del Deporte, 15 (2), 167-181.

Navas, L., Soriano, J. A., Holgado, F. P., \& López, M. (2009). Las orientaciones de meta de los estudiantes y los deportistas: perfiles motivacionales. Acción Psicológica, 6 (2), 17-29.

Nicholls, J. G. (1984a). Achievement motivation: conceptions of ability, subjective experience, task choice and performance. Psychological Review, 21 , 328-346. http://dx.doi.org/10.1037//0033295X.91.3.328

Nicholls, J. G. (1984b). Conceptions of ability and achievement motivation. En R. Ames \& C. Ames (Eds.), Research on motivation in education: Student motivation (pp. 318-332). Nueva York, NY: Academic Press.

Núñez, J. L., Martín-Albo, J. M., Paredes, A., Rodríguez, O., \& Chipana, N. (2011). The mediating role of perceived competente: testing a motivacional sequence in university students. Universitas Psychologica, 10 (3), 669-680.

Rivas, C., Romero, A., Pérez-Llantada, M. C., López, A., Pourtau, M., Molina, I., González, J., \& García-Mas, A. (2012). Bienestar psicológico, salud general, autonomía percibida y lesiones en futbolistas. Revista de Psicología del Deporte, 21 (2), 355-371.

Roberts, G. C. (2001). Understanding the dynamics of motivation in physical activity: The influence of achievement goals on motivational processes. En G. C. Roberts (Ed.), Advances in motivation in sport and exercise (pp. 1-50). Champaing, IL: Human Kinetics.

Ruiz, F., \& Piéron, M. (2013). Orientaciones de meta en Educación Física y nivel de actividad físico-deportiva en estudiantes mexicanos. Universitas Psychologica, 12 (1), 235-247. 
Sage, L., \& Kavussanu, M. (2007). Multiple goal orientations as predictors of moral behavior in youth soccer. The Sport Psychologist, 21 , 417-437.

Saies, E., Arribas-Galarrag, S., Cecchini, J. A., Luis-De-Cos, I., \& Otaegui, O. (2014). Diferencias en orientaciones de meta, motivación autodeterminada, inteligencia emocional y satisfacción con los resultados deportivos entre piragüistas expertos y novatos. Cuadernos de Psicología del Deporte, 14 (3), 21-30.

Sampeiro, J., Jiménez-Castuera, R., Lobato, S., Leyton, M., \& Claver, F. (2016). Variables motivacionales predictoras de las barreras para la práctica de ejercicio físico en adolescentes. Cuadernos de Psicología del Deporte, 16 (2), 65-75.

Sánchez-Oliva, D., Leo, F. M., Sánchez-Miguel, P. A., Amado, D., \& García-Calvo, T. (2010). Relación del clima motivacional creado por el entrenador con la motivación autodeterminada y la implicación hacia la práctica deportiva. International Journal of Sport Science, 6 (6), 177-195. http://dx.doi .org/10.5232/ricyde2010.02001

Saotome, H., Harada, K., \& Nakamura, Y. (2012). The relationship between change in perceived motivacional climate and change in goal orientations among japanese ice hockey players. International Journal of Sports Science and Coaching, 7 (1), 81-88.

Schwinger, M., \& Stiensmeier-Pelster, J. (2011). Performance-approach and performanceavoidance classroom goals and the adoption of personal achievement goals. British Journal of Educational Psychology, 81 , 680-699. http://dx.doi.org/10.1111/j.2 044-8279.2010.02012.x

Troncoso, S. M., Burgos, C. J., \& López-Walle, J. M. (2015). Climas motivacionales, liderazgo y cohesión grupal en contexto deportivo universitario. Educación Física y Ciencia, 17 (1), 1-12.

Valle, A., Rodríguez, S., Cabanach, R. G., Núñez, J.C., González-Pienda, J. A., \& Rosario, P. (2010). Perfiles motivacionales y diferencias en variables afectivas, motivacionales y de logro. Universitas Psychologica, 9 (1), 109-121.

Vanderberg, R. J., \& Lance, C. E. (2000). A review and synthesis of the measurement invariance literature: Suggestions, practices, and recommendations for organizational research. Organizational Research Methods, 3 , 4-70.

Wosnitza, M., \& Volet, S. (2012). Editorial introduction: Multiple goals in learning contexts. Applied Psychology: An International Review, 61 , 513-519. http://dx.doi.org/10.1111/j.14640597.2012.00503.x

Zarauz, A., \& Ruiz-Juan, F. (2014). Análisis de la motivación en el atletismo: un estudio con veteranos. Universitas Psychologica, 13 (2), 501-515. http://dx.doi.org/10.11144 /Javeriana.UPSY-2.amae

Zarauz, A., Ruiz-Juan, F., \& Flores-Allende, G. (2016). Modelos predictores de la motivación en corredores de fondo en ruta en función de sus hábitos y entrenamiento. Revista Iberoamericana de Psicología del Ejercicio y el Deporte, 11 (2), 185-192.

Zubiaur, M., \& Del Riego, M. (2015). Motivaciones e intereses de practicantes de BMX adolescentes: un estudio piloto. Retos , 27 (1), 109-113.

\section{Notas}

* Artículo de investigación 